\title{
Direct Bilirubin Is More Valuable than Total Bilirubin for Predicting Prognosis in Patients with Liver Cirrhosis
}

\author{
Han Ah Lee ${ }^{1}$, Joon Young Jung ${ }^{1}$, Young-Sun Lee ${ }^{1}$, Young Kul Jung ${ }^{1}$, Ji Hoon Kim¹, Hyonggin An², Hyung Joon \\ Yim¹, Yoon Tae Jeen ${ }^{1}$, Jong Eun Yeon ${ }^{1}$, Kwan Soo Byun ${ }^{1}$, Soon Ho Um¹, and Yeon Seok Seo ${ }^{1}$ \\ Departments of ${ }^{1}$ Internal Medicine and ${ }^{2}$ Biostatistics, Korea University College of Medicine, Seoul, Korea
}

See editorial on page 490.

\section{Article Info}

Received May 30, 2020

Revised August 23, 2020

Accepted September 6, 2020

Published online December 11, 2020

\section{Corresponding Author}

Yeon Seok Seo

ORCID https://orcid.org/0000-0003-4171-6331

E-mail drseo@koera.ac.kr

\section{Hyunggin An}

ORCID https://orcid.org/0000-0002-0566-758X

E-mail hyonggin@gmail.com

Han Ah Lee and Joon Young Jung contributed equally to this work as first authors.
Background/Aims: Most prognostic prediction models for patients with liver cirrhosis include serum total bilirubin (TB) level as a component. This study investigated prognostic performance of serum direct bilirubin (DB) and developed new DB level-based prediction models for cirrhosis.

Methods: A total of 983 hospitalized patients with liver cirrhosis were included. DB-Model for End-Stage Liver Disease (MELD) score was calculated using MELD score formula, with serum DB level replacing TB level.

Results: Mean age of study population was 56.1 years. Alcoholic liver disease was the most frequent underlying condition (471 patients, 47.9\%). Within 6 months, 144 patients (14.6\%) died or received liver transplantation due to severe liver dysfunction. The area under the receiver operating characteristic curve (AUROC) for prediction of 6-month mortality with DB level was significantly higher than that with TB level $(p<0.001)$. The AUROC of DB-MELD score for prediction of 6 -month mortality was significantly higher than that of MELD score $(p<0.001)$. Patients were randomly divided into training $(n=492)$ and validation $(n=491)$ cohorts. A new prognostic prediction model, "Direct Bilirubin, INR, and Creatinine" (DiBIC) score, was developed based on the most significant predictors of 6-month mortality. In training set, AUROC of DiBIC score for prediction of 6-month mortality was 0.892 , which was significantly higher than that of the MELD score $(0.875, p=0.017)$, but not different from that of DB-MELD score $(0.886, p=0.272)$. Similar results were observed in validation set.

Conclusions: New prognostic models, DB-MELD and DiBIC scores, have good prognostic performance in liver cirrhosis patients, outperforming other currently available models. (Gut Liver 2021;15:599-605)

Key Words: Direct bilirubin; Prognosis; Prediction model; Liver cirrhosis

\section{INTRODUCTION}

As the fifth leading cause of deaths worldwide, liver cirrhosis is a global public health problem. ${ }^{1}$ The necessity of stratifying risk in patients with cirrhosis has been underlined, because cirrhosis usually progresses toward various complications or death. ${ }^{2}$ Furthermore, as liver transplantation is the only definitive treatment for patients at high risk of mortality, prognosis prediction is important for decision making and allocating liver grafts. ${ }^{3,4}$ However, cirrhosis is an extremely heterogeneous disease comprising various factors $;{ }^{5-7}$ hence, predicting prognosis of patients with cirrhosis is challenging.
Many prognostic models have been proposed to predict outcome and stratify risk in patients with cirrhosis. In particular, serum bilirubin level represents hepatic synthetic and excretory function well; hence, most well-recognized prognostic models including Child-Pugh score and Model for End-stage Liver Disease (MELD) score have serum total bilirubin (TB) as a component. ${ }^{8,9}$ In liver cirrhosis, direct bilirubin (DB) level increases due to both intrahepatic cholestasis and decreased hepatic bilirubin clearance resulting from portal flow distortion. ${ }^{10,11}$ Meanwhile, portosystemic shunting and splenomegaly provoke hemolysis, leading to an increase in indirect bilirubin level. ${ }^{12,13}$

Because increases in DB and indirect bilirubin levels 
have different pathophysiologies, ${ }^{14,15}$ patients with predominant indirect bilirubinemia may have different predisposing factors and prognosis than patients with direct bilirubinemia. However, to our knowledge, few studies have addressed differences in prognostic performance between TB level and DB level in liver cirrhosis. In the present study, we hypothesized that DB level may have more beneficial role than TB level in predicting prognosis of cirrhotic patients, because indirect bilirubinemia results from other causes rather than impaired hepatic function itself. Accordingly, we compared the prognostic performance of DB level with that of TB level, and aimed to develop new serum DB level-based prognostic model that would be more useful than previous models in predicting prognosis in patients with liver cirrhosis.

\section{MATERIALS AND METHODS}

\section{Subjects}

A total of 2,891 patients with liver cirrhosis who were hospitalized in Korea University Anam Hospital between 2004 and 2018 were considered eligible (Fig. 1). Liver cirrhosis was diagnosed by either clinical or histological evaluation, when typical ultrasonographic findings were found together with low platelet count $(<100,000 / \mu \mathrm{L})$, varices, or overt complication of liver cirrhosis. ${ }^{16}$ The exclusion criteria included the following: (1) age < 18 years; (2) insufficient follow-up period ( $<6$ months); (3) insufficient clinical or laboratory information; (4) history of hepatocellular carcinoma or organ transplantation (5) use of immunosuppressive agents; or (6) other significant medical illness.

This study protocol was consistent with the ethical guidelines of the Declaration of Helsinki and was approved by the Institutional Review Board of Korea University Anam Hospital (IRB number: 2018AN0381). The requirement for informed consent was waived due to the retrospective nature of the study.

\section{Data collection}

We investigated the patients' age, sex, international normalized ratio (INR), serum TB and DB, and creatinine levels at the time of admission. MELD score was calculated as previously described. ${ }^{9}$ We also calculated DB-MELD score, replacing TB level with $\mathrm{DB}$ level, using the following equation:

DB-MELD score $=9.57 \times \log _{\mathrm{e}}$ (serum creatinine level, $\mathrm{mg} / \mathrm{dL})+3.78 \times \log _{\mathrm{e}}([$ serum direct bilirubin level, $\mathrm{mg} / \mathrm{dL}]$ $+1)+11.2 \times \log _{\mathrm{e}}(\mathrm{INR})+6.43$. The discriminant limit for INR and serum creatinine was 1.0. DB level was adjusted by adding 1 , because it has a relatively lower numerical value.

\section{Outcomes}

The primary endpoint was 6-month liver dysfunctionrelated mortality or liver transplantation. The follow-up period was calculated from the index date to the date of mortality, liver transplantation, or last follow-up.

\section{Statistical analysis}

Quantitative variables were presented as means \pm standard deviation and compared using the Student t-test or MannWhitney $\mathrm{U}$ test, whereas categorical variables were presented as numbers (\%) and compared using the chi-square test or Fisher exact test. Overall survival was defined as the time interval between initial hospitalization and liver-related death or transplantation. Survival curves were estimated using the Kaplan-Meier method and compared using the log-rank test. Univariate and multivariate Cox regression analyses were performed on the training cohort to evaluate

2,891 Patients with liver cirrhosis hospitalized in

Korea University Anam Hospital between 2004 and 2018

\begin{tabular}{|l|l|}
\hline & Exclusion criteria \\
105 Age <18 years \\
304 Insufficient follow-up period (<6 months) \\
453 Insufficient clinical or laboratory information \\
498 History of hepatocellular carcinoma or organ transplant \\
145 Use of immunosuppressive agents \\
245 Other significant medical illness \\
158 Non-liver-related death \\
\cline { 2 - 2 }
\end{tabular}

983 Patients were finally selected for statistical analysis

492 Training set $(50.1 \%)$
491 Validation set $(49.9 \%)$
Fig. 1. Flowchart of the patient population. 
the influence of variables on survival. New predictive model was established using significant predictors and their beta coefficients. The predictive performance of each model for 6-month mortality were assessed using area under the receiver operating characteristic curve (AUROC) and 95\% confidence intervals (CIs). The AUROCs of the prediction models were compared using the Hanley-McNeil test. ${ }^{17}$ Statistical analyses were conducted using IBM SPSS Statistics software version 23.0 (IBM Corp., Armonk, NY, USA). Two-sided $\mathrm{p}$-values $<0.05$ were considered significant.

\section{RESULTS}

\section{Baseline characteristics of the study population}

A total of 983 hospitalized patients with liver cirrhosis were finally subjected to statistical analysis (Fig. 1). Six hundred and eighty patients (69.2\%) had decompensated cirrhosis. The cause of admission was acute decompensation $(385,39.2 \%)$, elevation of TB level $(224,22.8 \%)$, infection (253, 25.7\%), liver biopsy (23, 2.3\%), and others (98, $10.0 \%)$. Among these, 144 patients (14.6\%) either died or received liver transplantation due to severe liver dysfunction within 6 months. The causes of 6 -month mortality were liver failure ( 85 patients, $58.7 \%$ ), varix bleeding (23 patients, $15.9 \%$ ), and sepsis (36 patients, $25.4 \%$ ). The baseline characteristics of entire patients are presented in Table
1. When compared to patients without 6-month mortality, INR, serum TB, DB, and creatinine levels, MELD score and DB-MELD score were significantly higher, while serum sodium level was significantly lower in patients with 6-month mortality (all p<0.001).

\section{Comparison of predictive performance for 6-month mortality between TB and DB; and between MELD score and DB-MELD score}

The AUROC of DB level for predicting 6-month mortality $(0.843 ; 95 \% \mathrm{CI}, 0.811$ to 0.875$)$ was significantly higher than that of TB level $(0.818 ; 95 \% \mathrm{CI}, 0.781$ to 0.856$)$ (difference between areas, $0.025 ; 95 \% \mathrm{CI}, 0.014$ to 0.037 ; $\mathrm{z}$ statistic, 4.326; $\mathrm{p}<0.001$ ) (Fig. 2A). The AUROC of DBMELD score for predicting 6-month mortality $(0.877 ; 95 \%$ CI, 0.847 to 0.907$)$ was significantly higher than that of MELD score (0.866; $95 \%$ CI, 0.834 to 0.898 ) (difference between areas, 0.012 ; $95 \%$ CI, 0.005 to 0.018 ; z statistic, 3.372; $\mathrm{p}<0.001$ ) (Fig. 2B).

\section{Baseline characteristics of training and validation datasets}

To develop the new prognosis prediction model based on DB level, patients were randomly assigned to the training $(\mathrm{n}=492,50.1 \%)$ and validation datasets $(\mathrm{n}=491,49.9 \%)$ by computer-generated randomized number (Fig. 1). The baseline characteristics of the patients from each dataset

Table 1. Baseline Characteristics of the Study Population and Comparison between Surviving and Nonsurviving Patients

\begin{tabular}{|c|c|c|c|c|}
\hline Variable & All patients ( $n=983$ ) & $\begin{array}{l}\text { Patients without 6-month } \\
\text { mortality ( } n=839,85.4 \%)\end{array}$ & $\begin{array}{l}\text { Patients with 6-month } \\
\text { mortality ( } n=144,14.6 \%)\end{array}$ & $\mathrm{p}$-value \\
\hline \multicolumn{5}{|l|}{ Demographic variables } \\
\hline Age, yr & $56.1 \pm 11.9$ & $56.1 \pm 11.9$ & $56.4 \pm 12.1$ & 0.769 \\
\hline Follow-up duration, mo & $28.0 \pm 21.7$ & $32.6 \pm 29.2$ & $1.7 \pm 1.4$ & $<0.001$ \\
\hline Male sex & 701 (71.3) & $604(72.0)$ & $97(67.4)$ & 0.257 \\
\hline Hypertension & 305 (31.0) & 263 (31.3) & $42(29.2)$ & 0.601 \\
\hline Diabetes & 326 (33.2) & $284(33.8)$ & 42 (29.2) & 0.270 \\
\hline Etiology & & & & 0.035 \\
\hline Hepatitis B virus & 315 (32.0) & 284 (33.8) & $31(21.5)$ & \\
\hline Hepatitis C virus & $63(6.4)$ & $52(6.2)$ & $11(7.6)$ & \\
\hline Alcohol & $471(47.9)$ & $392(46.7)$ & 79 (54.9) & \\
\hline Others & $134(13.6)$ & $111(13.2)$ & $23(16.0)$ & \\
\hline \multicolumn{5}{|l|}{ Laboratory variables } \\
\hline Platelet count, $\times 10^{9} / \mathrm{L}$ & $98.9 \pm 59.0$ & $99.2 \pm 57.0$ & $97.2 \pm 69.8$ & 0.709 \\
\hline Prothrombin time, INR & $1.5 \pm 0.5$ & $1.5 \pm 0.5$ & $2.0 \pm 0.7$ & $<0.001$ \\
\hline Serum albumin, g/dL & $3.2 \pm 0.7$ & $3.3 \pm 0.7$ & $2.8 \pm 0.5$ & $<0.001$ \\
\hline Total bilirubin, mg/dL & $5.9 \pm 6.9$ & $4.5 \pm 4.4$ & $14.0 \pm 11.7$ & $<0.001$ \\
\hline Direct bilirubin, mg/dL & $3.0 \pm 4.4$ & $2.1 \pm 2.5$ & $8.5 \pm 7.9$ & $<0.001$ \\
\hline Alanine aminotransferase, IU/L & $87.3 \pm 234.3$ & $85.8 \pm 241.9$ & $96.0 \pm 183.9$ & 0.629 \\
\hline Creatinine, $\mathrm{mg} / \mathrm{dL}$ & $1.0 \pm 0.6$ & $0.9 \pm 0.3$ & $1.4 \pm 1.3$ & $<0.001$ \\
\hline Sodium, $\mathrm{mmol} / \mathrm{L}$ & $136.0 \pm 7.4$ & $136.5 \pm 7.5$ & $132.9 \pm 6.1$ & $<0.001$ \\
\hline MELD score & $10.8 \pm 2.8$ & $10.2 \pm 2.2$ & $14.4 \pm 3.4$ & $<0.001$ \\
\hline DB-MELD score & $10.4 \pm 2.6$ & $9.8 \pm 1.9$ & $13.9 \pm 3.2$ & $<0.001$ \\
\hline
\end{tabular}

Data are presented as mean \pm SD or number (\%).

INR, international normalized ratio; DB-MELD, direct bilirubin Model for End-Stage Liver Disease. 

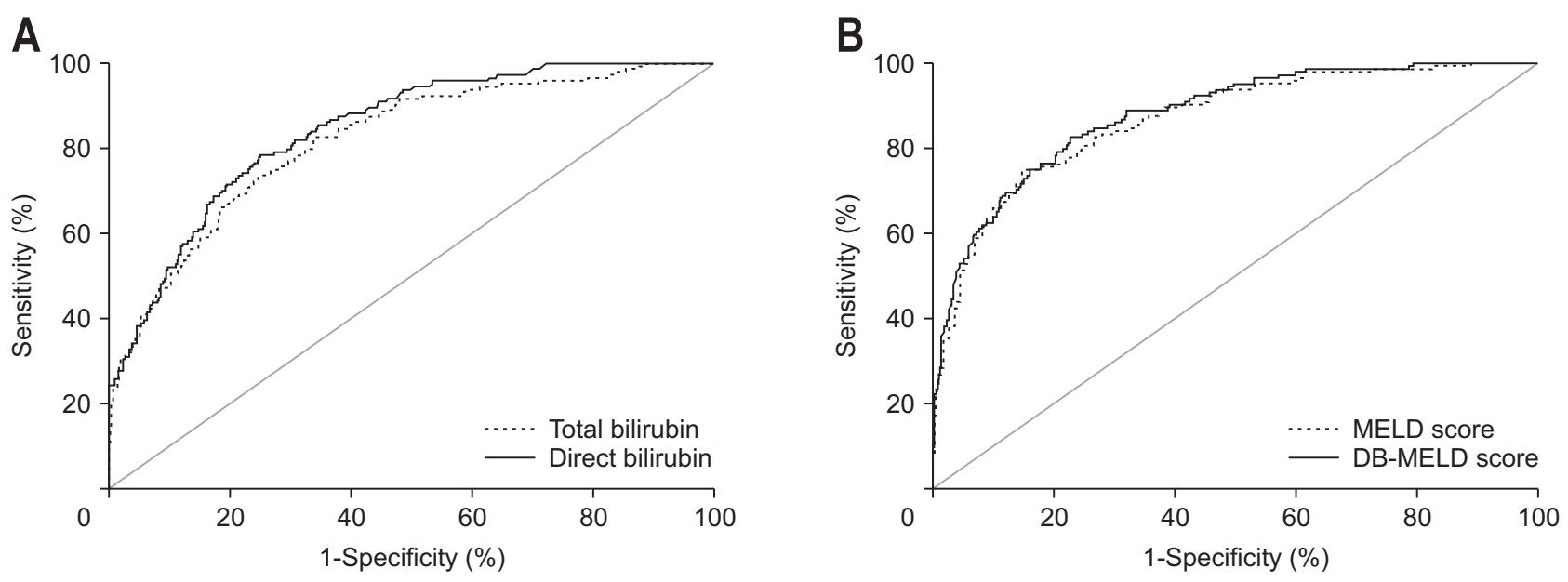

Fig. 2. The receiver operating characteristic curves for the prediction of 6-month mortality with (A) total bilirubin and direct bilirubin; (B) the MELD and DB-MELD scores in patients with cirrhosis.

MELD, Model for End-Stage Liver Disease; DB, direct bilirubin.

Table 2. Predictors of 6-Month Mortality in the Training Set (n=492)

\begin{tabular}{|c|c|c|c|c|}
\hline \multirow{2}{*}{ Variable } & \multicolumn{2}{|c|}{ Univariate analysis } & \multicolumn{2}{|c|}{ Multivariate analysis } \\
\hline & $\operatorname{HR}(95 \% \mathrm{Cl})$ & $\mathrm{p}$-value & $\operatorname{HR}(95 \% \mathrm{Cl})$ & $\mathrm{p}$-value \\
\hline Age, yr & 0.996 (0.975-1.017) & 0.699 & & \\
\hline Male sex & 0.884 (0.508-1.537) & 0.663 & & \\
\hline Hepatitis B virus & $2.319(1.230-4.373)$ & 0.009 & $1.690(0.767-3.714)$ & 0.193 \\
\hline Diabetes & 1.059 (0.622-1.803) & 0.834 & & \\
\hline Hypertension & $1.135(0.662-1.946)$ & 0.646 & & \\
\hline Platelet count, $\times 10^{9} / \mathrm{L}$ & $1.000(0.996-1.004)$ & 0.999 & & \\
\hline Prothrombin time, INR & 5.905 (3.519-9.910) & $<0.001$ & 2.670 (1.509-4.723) & 0.001 \\
\hline Serum albumin, g/dL & $0.282(0.175-0.453)$ & $<0.001$ & $0.552(0.293-1.040)$ & 0.066 \\
\hline Total bilirubin, mg/dL & $1.180(1.134-1.227)$ & $<0.001$ & $0.941(0.830-1.067)$ & 0.341 \\
\hline Direct bilirubin, $\mathrm{mg} / \mathrm{dL}$ & $1.334(1.241-1.433)$ & $<0.001$ & $1.373(1.114-1.694)$ & 0.003 \\
\hline Alanine aminotransferase, IU/L & $1.000(0.998-1.001)$ & 0.646 & & \\
\hline Creatinine, $\mathrm{mg} / \mathrm{dL}$ & $5.692(3.022-10.720)$ & $<0.001$ & $3.271(1.423-7.516)$ & 0.005 \\
\hline Sodium, $\mathrm{mmol} / \mathrm{L}$ & $0.872(0.833-0.914)$ & $<0.001$ & $0.941(0.885-1.000)$ & 0.049 \\
\hline
\end{tabular}

INR, international normalized ratio; $\mathrm{HR}$, hazards ratio; $\mathrm{Cl}$, confidence interval.

are presented in Supplementary Table 1. There were no significant differences in demographic or laboratory variables between the two datasets, including MELD score (10.9 vs 10.8, $\mathrm{p}=0.595)$ and DB-MELD score (10.4 vs $10.4, \mathrm{p}=0.703$ ).

\section{1) Training set}

Univariate and multivariate analyses were performed in the training set to reveal the predictors of 6-month mortality (Table 2). On multivariate analysis, prolonged INR (hazard ratio $[\mathrm{HR}]=2.670)$, higher $\mathrm{DB}$ level $(\mathrm{HR}=1.373)$, and higher creatinine level $(\mathrm{HR}=3.271)$ independently predicted an increased risk of 6-month mortality, whereas a higher sodium level $(\mathrm{HR}=0.941)$ independently predicted a decreased risk of 6-month mortality (all $\mathrm{p}<0.05$ ). To develop the new prognosis prediction model, binary regression analysis was performed using the most potent predictors: DB, INR, and creatinine level (Table 3). Using the beta coefficients of the predictors, new prognosis prediction model, the "Direct Bilirubin, INR, and Creatinine" (DiBIC) score was established as follows:

DiBIC score $=4.712 \times \log _{\mathrm{e}}(\mathrm{INR})+3.584 \times \log _{\mathrm{e}}([$ serum DB level, $\mathrm{mg} / \mathrm{dL}]+1)+6.918 \times \log _{\mathrm{e}}$ (serum creatinine level, $\mathrm{mg} /$ $\mathrm{dL})$. The discriminant limit for INR and serum creatinine was 1.0. DB level was adjusted by adding 1 , because it has a relatively lower numerical value.

The mean DiBIC score was 2.7, and it was significantly higher in patients with 6-month mortality than in those without (5.4 vs 2.3, $\mathrm{p}<0.001$ ). The AUROC of DiBIC score for predicting 6-month mortality $(0.892$; $95 \% \mathrm{CI}, 0.861$ to 0.918 ), was significantly higher than that of MELD score (0.875; 95\% CI, 0.843 to 0.903$)$ (difference between areas, 0.017; $95 \% \mathrm{CI}, 0.003$ to 0.031 ; z statistic, 2.386; $\mathrm{p}=0.017$ ), while it did not differ with that of DB-MELD score (0.886; $\mathrm{p}=0.272$ ). The AUROC of DB-MELD score was signifi- 
Table 3. Binary Regression Analysis for 6-Month Mortality in the Training Set (n=492)

\begin{tabular}{lccc}
\hline \multicolumn{1}{c}{ Variable } & Beta coefficient & OR (95\% Cl) & p-value \\
\hline Prothrombin time, INR & 4.712 & $111.220(9.015-1,372.144)$ & $<0.001$ \\
Direct bilirubin, mg/dL & 3.584 & $36.020(12.246-105.942)$ & $<0.001$ \\
Creatinine, mg/dL & 6.918 & $1,010.602(25.671-39,785.517)$ & $<0.001$ \\
\hline
\end{tabular}

INR, international normalized ratio; $\mathrm{OR}$, odds ratio; $\mathrm{Cl}$, confidence interval.
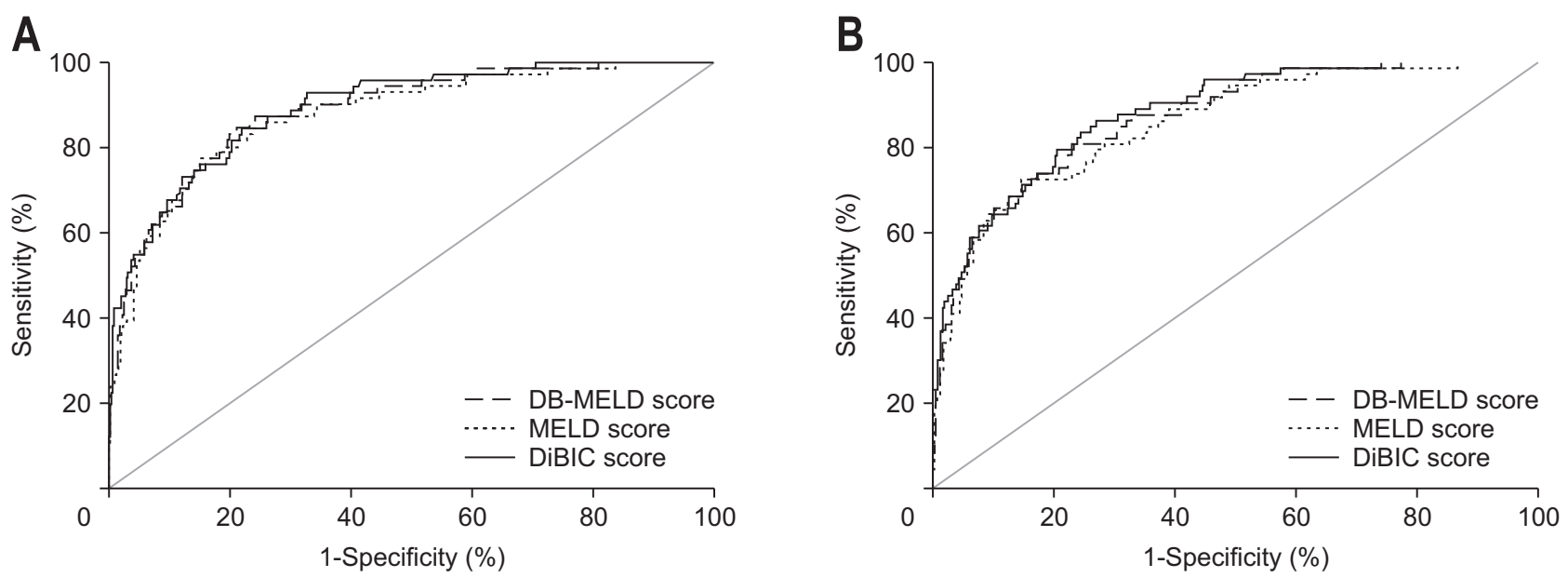

Fig. 3. The receiver operating characteristic curves for the prediction of 6-month mortality with the MELD and DB-MELD scores in the training set (A) and the validation set (B).

MELD, Model for End-Stage Liver Disease; DB, direct bilirubin; INR, international normalized ratio; DiBIC, Direct Bilirubin, INR, and Creatinine.

cantly higher than that of MELD score (difference between areas, $0.011 ; 95 \%$ CI, 0.001 to 0.021 ; z statistic, 2.108; $\mathrm{p}=0.035)$ (Fig. 3A).

\section{2) Validation set}

The mean DiBIC score among patients in the validation group was 2.7 , and it was significantly higher in patients with 6-month mortality than in those without (4.9 vs $2.3, \mathrm{p}<0.001)$. The AUROC of DiBIC score for predicting 6-month mortality was 0.880 (95\% CI, 0.848 to 0.907 ), which was significantly higher than that of MELD score (0.857; 95\% CI, 0.822 to 0.886$)$ (difference between areas, 0.023 ; $95 \% \mathrm{CI}, 0.009$ to 0.037 ; $\mathrm{z}$ statistic, 3.217 ; $\mathrm{p}=0.001$ ) and that of DB-MELD score $(0.869 ; 95 \%$ CI, 0.836 to 0.897 ; difference between areas, $0.011 ; 95 \% \mathrm{CI}, 0.001$ to $0.022 ; \mathrm{z}$ statistic, 2.032; $\mathrm{p}=0.042$ ). The AUROC value of DB-MELD score was significantly higher than that of MELD score (difference between areas, 0.012; 95\% CI, 0.003 to $0.021 ; \mathrm{z}$ statistic, 2.591; $\mathrm{p}=0.010$ ) (Fig. 3B).

\section{Priority for liver transplantation according to the MELD score and DiBIC score}

To evaluate the impact of DiBIC score in clinical decision making, DiBIC score of 50 patients with highest MELD score was calculated. The priority for liver transplantation was quite different between MELD and DiBIC score-based allocation system (Supplementary Table 2).

\section{DISCUSSION}

Various prediction models have been developed and adopted to predict outcome and stratify risk in patients with cirrhosis, including Child-Pugh score and MELD score. These well-established models can be useful in predicting risk in patients with liver cirrhosis, but they may not be sufficient. In the present large retrospective cohort study, we revealed that DB level had higher performance than TB level in predicting 6-month survival in patients with cirrhosis $(\mathrm{p}<0.001)$. In both the training and validation cohorts, DB-MELD score, in which DB level replaces TB level in calculating MELD score, had better performance than MELD score in terms of 6-month mortality prediction (all $\mathrm{p}<0.05$ ). Furthermore, we developed and validated a new prognosis prediction model: DiBIC score, which uses the most significant predictors of 6-month mortality in patients with cirrhosis (INR, DB and creatinine level). DiBIC score consistently outperformed MELD score in both training and validation cohorts (all $\mathrm{p}<0.05$ ).

The present study has several strengths and implications. First, we evaluated the performance of DB level as a potent predictor of prognosis in patients with liver cirrhosis. We found that DB level and DB-MELD score outperformed TB level and MELD score, respectively (all $\mathrm{p}<0.05$ ), as we hypothesized. This result suggests that intrahepatic cholestasis and decreased hepatic clearance play a more 
critical role than hemolysis provoked by splenomegaly in the survival of patients with liver cirrhosis. In addition, glucuronyl conjugation of indirect bilirubin is also impaired in decompensated cirrhosis, prompting increase in indirect bilirubin level. However, the clinical impact of impairment of bilirubin conjugation in prognosis of patients with cirrhosis is not well known. Therefore, TB level itself has many limitations in predicting prognosis in patients with liver cirrhosis. It is supported by previous study conducted by López-Velázquez et al., ${ }^{18}$ which suggested that DB level had higher prognostic value than TB level in predicting 1-week mortality in patients with cirrhosis and acute-on-chronic liver failure (AUROC value 0.751 vs 0.746; $\mathrm{p}<0.05$ ). However, because the exact mechanisms were not clarified, further in vitro studies are needed to confirm this hypothesis.

Second, we developed and evaluated a new, novel prognostic model, the DiBIC score, with DB level. DiBIC score had better prognostic value than MELD score in both the training and validation cohorts (all $\mathrm{p}<0.05$ ). DiBIC score also has INR and serum creatinine level as its other components, which were the most potent predictors of survival in the binary regression analysis. Because DiBIC score includes these objective parameters that reflect the deterioration of patients with liver cirrhosis, reproducible prediction of short-term mortality was possible.

Additionally, we reweighed the components according to the predictive impact of each, updating the formula with different coefficients to improve the predictive value of DiBIC score. Specifically, DiBIC score assigned lower weights to INR and serum creatinine, and higher weight to DB; a similar refinement of MELD score which performed better than the existing MELD score in predicting prognosis. ${ }^{19-21}$ Although renal dysfunction is a major risk factor for mortality in liver cirrhosis, serum creatinine level is frequently overweighed in MELD score. For example, in same MELD score, patients with severely impaired renal function and less severe liver dysfunction have better prognosis than patients with mild renal dysfunction and more severe liver dysfunction. Furthermore, serum creatinine level is frequently overestimated because patients with liver cirrhosis have decreased muscle mass. ${ }^{22,23}$ Therefore, reweighing of each variable improved the predictive performance of DiBIC score.

We are also aware of several issues that remain unresolved. First, the present study may have suffered from selection bias because it had a retrospective design. Moreover, although the study had a large sample size and underwent internal validation, future studies are needed to implement external validation of these results. Secondly, although we developed and validated the prognostic value of DB level and DB-based prediction models in wide range of patients with liver cirrhosis, we could not confirm the clinical implication of these values in the survival of patients with compensated cirrhosis, because only one patient with compensated cirrhosis died within the 6-month study period. DiBIC score may be insufficient when applied to compensated patients because, like MELD score, DiBIC score fails to account for significant predictors related to portal hypertension or complications of liver cirrhosis. ${ }^{24}$ Lastly, to better refine and replace the existing MELD score, DiBIC score should be validated over various observational periods and in a broad spectrum of liver diseases. MELD score has been shown to be reliable in predicting 3-month, 1-year, and 5-year survival across a wide range of liver diseases. ${ }^{25-28}$ Further studies should evaluate DiBIC score in the classification of other liver diseases, such as acute-on-chronic liver failure and acute hepatitis, with various observational periods before the model can be put to wider clinical use.

In conclusion, we showed that DB level predicted prognosis better than TB level in patients with liver cirrhosis. In addition, DB-based prediction models (DB-MELD and DiBIC score) could be implemented to refine and replace MELD score in patients with liver cirrhosis. These new prediction models could be beneficial and helpful to guide clinical decision making including transplant allocation.

\section{CONFLICTS OF INTEREST}

No potential conflict of interest relevant to this article was reported.

\section{AUTHOR CONTRIBUTIONS}

Conception and design: Y.S.S. Development of methodology: Y.S.S. Acquisition, analysis and interpretation of data: Y.S.S., J.Y.J., H.A.L. Writing, review, and/or revision of the manuscript: H.A.L., J.Y.J., Y.S.L., Y.K.J., J.H.K., H.A., H.J.Y., Y.T.J., J.E.Y., K.S.B., S.H.U., Y.S.S. Administrative, technical, or material support: H.A., Y.S.S. Study supervision: Y.S.S.

\section{ORCID}

Han Ah Lee Joon Young Jung Young-Sun Lee Young Kul Jung Ji Hoon Kim Hyonggin An https://orcid.org/0000-0003-4082-1121 https://orcid.org/0000-0003-4579-8709 https://orcid.org/0000-0001-6396-0859 https://orcid.org/0000-0002-6566-1382 https://orcid.org/0000-0003-3924-0434 https://orcid.org/0000-0002-0566-758X 
Hyung Joon Yim https://orcid.org/0000-0002-6036-2754

Yoon Tae Jeen

Jong Eun Yeon

Kwan Soo Byun

Soon Ho Um

Yeon Seok Seo https://orcid.org/0000-0003-0220-3816

https://orcid.org/0000-0002-0510-7371

https://orcid.org/0000-0003-4031-842X

https://orcid.org/0000-0002-6390-2218

https://orcid.org/0000-0003-4171-6331

\section{REFERENCES}

1. Scaglione S, Kliethermes S, Cao G, et al. The epidemiology of cirrhosis in the United States: a population-based study. J Clin Gastroenterol 2015;49:690-696.

2. Kim HJ, Lee HW. Important predictor of mortality in patients with end-stage liver disease. Clin Mol Hepatol 2013;19:105-115.

3. Lee HA, Cho EY, Kim TH, et al. Risk factors for dropout from the liver transplant waiting list of hepatocellular carcinoma patients under locoregional treatment. Transplant Proc 2018;50:3521-3526.

4. Korean Association for the Study of the Liver (KASL). KASL clinical practice guidelines for liver cirrhosis: ascites and related complications. Clin Mol Hepatol 2018;24:230-277.

5. Gu DH, Kim MY, Seo YS, et al. Clinical usefulness of psoas muscle thickness for the diagnosis of sarcopenia in patients with liver cirrhosis. Clin Mol Hepatol 2018;24:319-330.

6. Lee SK, Song MJ, Kim SH, Ahn HJ. Cardiac diastolic dysfunction predicts poor prognosis in patients with decompensated liver cirrhosis. Clin Mol Hepatol 2018;24:409-416.

7. D’Amico G, Garcia-Tsao G, Pagliaro L. Natural history and prognostic indicators of survival in cirrhosis: a systematic review of 118 studies. J Hepatol 2006;44:217-231.

8. Child CG, Turcotte JG. Surgery and portal hypertension. Major Probl Clin Surg 1964;1:1-85.

9. Kamath PS, Wiesner RH, Malinchoc M, et al. A model to predict survival in patients with end-stage liver disease. Hepatology 2001;33:464-470.

10. Zieve L. Jaundice in cirrhosis. JAMA 1965;191:475-479.

11. Ohkubo A. Bilirubin metabolism in liver cirrhosis. Nihon Rinsho 1994;52:138-144.

12. Felsher BF, Redeker AG, Reynolds TB. Indirect reacting hyperbilirubinemia in cirrhosis: its relation to red cell survival. Am J Dig Dis 1968;13:598-607.

13. Aravinthan AD, Alexander GJ. Hepatocyte senescence explains conjugated bilirubinaemia in chronic liver failure. J Hepatol 2015;63:532-533.

14. Trauner M, Meier PJ, Boyer JL. Molecular pathogenesis of cholestasis. N Engl J Med 1998;339:1217-1227.

15. Vítek L, Ostrow JD. Bilirubin chemistry and metabolism; harmful and protective aspects. Curr Pharm Des

2009;15:2869-2883.

16. Jung KS, Kim SU, Ahn SH, et al. Risk assessment of hepatitis $B$ virus-related hepatocellular carcinoma development using liver stiffness measurement (FibroScan). Hepatology 2011;53:885-894.

17. Hanley JA, McNeil BJ. A method of comparing the areas under receiver operating characteristic curves derived from the same cases. Radiology 1983;148:839-843.

18. López-Velázquez JA, Chávez-Tapia NC, Ponciano-Rodríguez $\mathrm{G}$, et al. Bilirubin alone as a biomarker for short-term mortality in acute-on-chronic liver failure: an important prognostic indicator. Ann Hepatol 2013-2014;13:98-104.

19. Leise MD, Kim WR, Kremers WK, Larson JJ, Benson JT, Therneau TM. A revised model for end-stage liver disease optimizes prediction of mortality among patients awaiting liver transplantation. Gastroenterology 2011;140:1952-1960.

20. Kim WR, Biggins SW, Kremers WK, et al. Hyponatremia and mortality among patients on the liver-transplant waiting list. N Engl J Med 2008;359:1018-1026.

21. Sharma P, Schaubel DE, Sima CS, Merion RM, Lok AS. Reweighting the model for end-stage liver disease score components. Gastroenterology 2008;135:1575-1581.

22. Kim TH, Lee HA, Seo YS, et al. Assessment and prediction of acute kidney injury in patients with decompensated cirrhosis with serum cystatin $\mathrm{C}$ and urine $\mathrm{N}$-acetyl- $\beta-\mathrm{D}$ glucosaminidase. J Gastroenterol Hepatol 2019;34:234-240.

23. Yoo JJ, Kim SG, Kim YS, et al. Estimation of renal function in patients with liver cirrhosis: impact of muscle mass and sex. J Hepatol 2019;70:847-854.

24. Peng Y, Qi X, Guo X. Child-Pugh versus MELD score for the assessment of prognosis in liver cirrhosis: a systematic review and meta-analysis of observational studies. Medicine (Baltimore) 2016;95:e2877.

25. Said A, Williams J, Holden J, et al. Model for end stage liver disease score predicts mortality across a broad spectrum of liver disease. J Hepatol 2004;40:897-903.

26. Dickson ER, Grambsch PM, Fleming TR, Fisher LD, Langworthy A. Prognosis in primary biliary cirrhosis: model for decision making. Hepatology 1989;10:1-7.

27. Testa R, Testa E, Giannini E, et al. Trans-catheter arterial chemoembolisation for hepatocellular carcinoma in patients with viral cirrhosis: role of combined staging systems, Cancer Liver Italian Program (CLIP) and Model for End-stage Liver Disease (MELD), in predicting outcome after treatment. Aliment Pharmacol Ther 2003;17:1563-1569.

28. Huo TI, Lin HC, Hsia CY, et al. The model for end-stage liver disease based cancer staging systems are better prognostic models for hepatocellular carcinoma: a prospective sequential survey. Am J Gastroenterol 2007;102:1920-1930. 